\title{
The Six-Minute Walking Exercise On The Fatigue Levels In Hemodialysis Patients
}

\author{
Yohanes Gamayana Trimawang $\mathrm{Aji}^{{ }^{*}}$, Krisna Yetti ${ }^{2}$, Lestari Sukmarini ${ }^{3}$ \\ ${ }_{1}^{1}$ Academy of PGI Cikini Hospital, Central Jakarta, Indonesia \\ 2 Faculty of Nursing University of Indonesia, Depok, Indonesia \\ ${ }^{3}$ Faculty of Nursing University of Indonesia, Depok, Indonesia \\ a yohanes.gamayana@akperrscikini.ac.id \\ * coresponding author
}

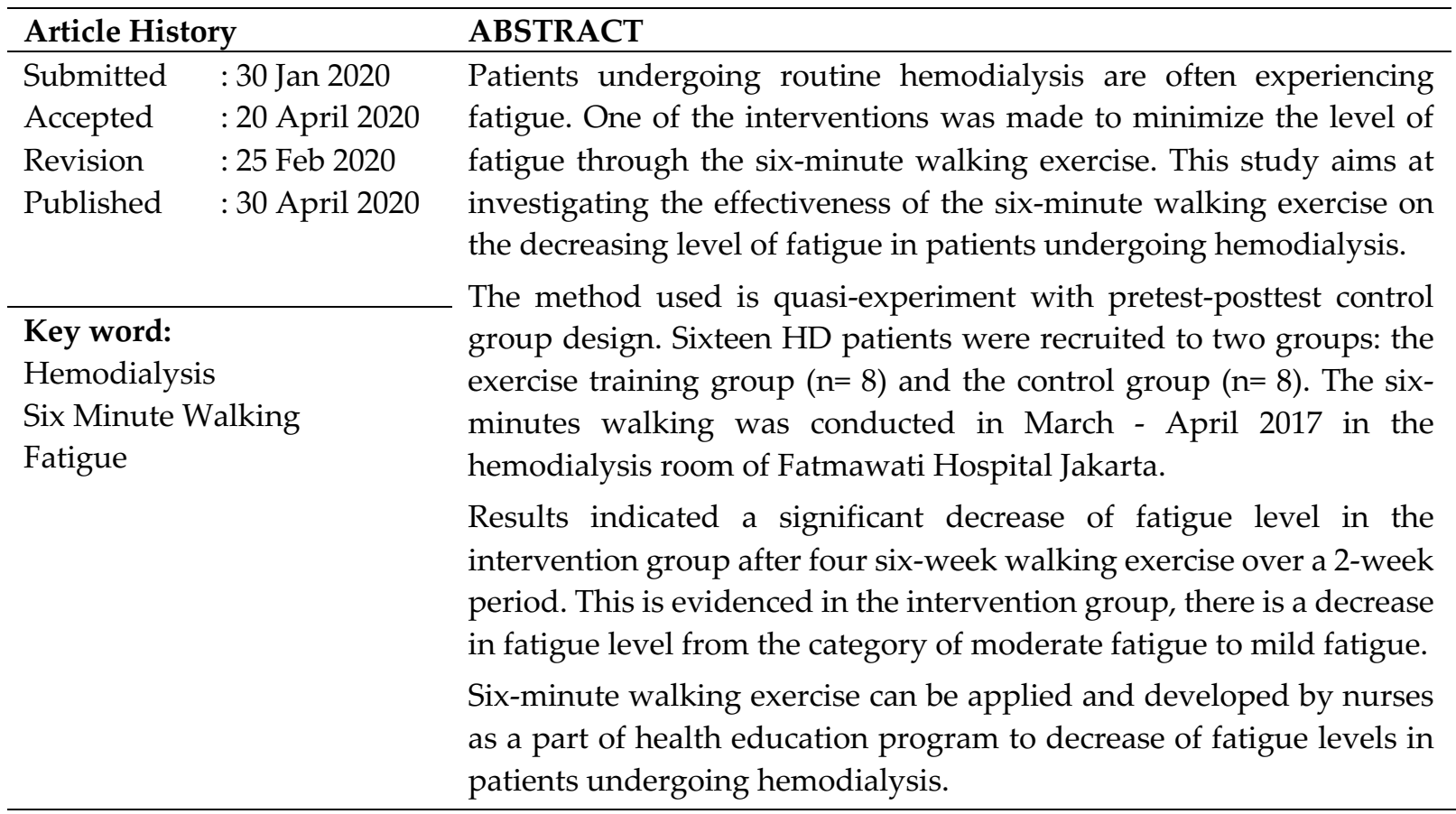

This is an openaccess article under the CC-BY-SAlicense.
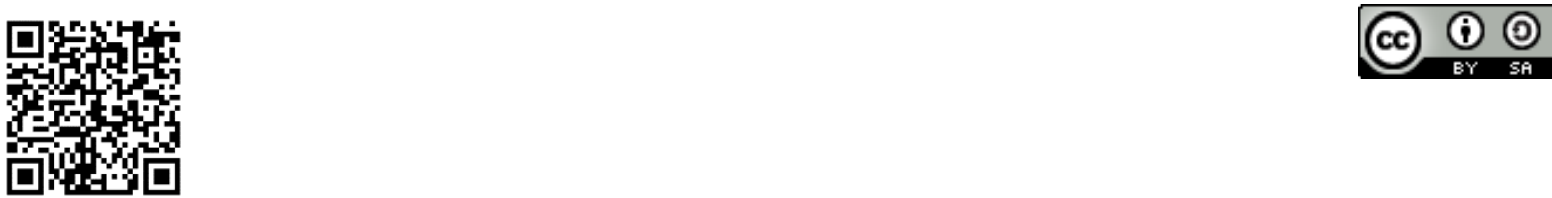

\section{Introduction}

Hemodialysis is one treatment for patients with chronic renal failure to prolong their life. Hemodialysis is a procedure of cleansing blood through an artificial kidney and assisted its execution using a machine. Hemodialysis cannot cure or recover kidney disease and is unable to offset the loss of metabolic or endocrine activity performed by the kidneys and the impact of renal failure and its therapy. ${ }^{1}$

The most common symptom in patients undergoing hemodialysis is fatigue (fatigue). Fatigue is a major complaint in patients undergoing long-term hemodialysis. According to Nanda (2009), fatigue is an overwhelming sense of fatigue and a decrease in the capacity of physical and mental work at a constant level. ${ }^{2}$ Fatigue is a condition in which an individual experiences a feeling of fatigue and a decrease in physical and mental ability and also a 
decrease of rest. The greatest impact of fatigue if left untreated can affect the quality of life and cause increased dependence on others, weakness, physical and mental decline, social withdrawal and depression.

One study of hemodialysis patient fatigue was that physical fatigue occupies the highest score. ${ }^{3}$ McCann and Boore (2000) found that 40 hemodialysis patients experiencing fatigue related to sleep disorders, depression, anxiety, decreased physical ability, feeling of laziness, muscle weakness and pain in the joints. ${ }^{4}$

The role of nurses in overcoming fatigue is to examine carefully the level of fatigue, the number of activities that can be done and the appropriate intervention for the patients. There are four strategies to lower the fatigue, such as: exercises, behavioral strategies, nutritional support and physiological approaches. One of exercises that can be performed is walking exercises for six-minutes or more known walking.

The six-minute walking is a rhythmic gait with the arms swinging in the rhythm of a person's path for six minutes. This action serves to control the balance, increase the effectiveness of the heart pump, improve circulation, endurance, mobility level, quality of life, peripheral tissue and heart. The results suggest that walking was effective in reducing the fatigue level of hemodialysis patients characterized by decreased frequency and intensity of leg muscle cramps. ${ }^{5}$

\section{Material and method}

The method used is quasi-experiment with pretest-posttest control group design. Sixteen participants divided into 2 groups, 8 patients were selected for intervention group and the remaining 8 patients for control group. The intervention group received six-minute walking exercises, while the control group did not get exercise only usual care.

The total participants in this study were 16 patients undergoing hemodialysis at Fatmawati Hospital Jakarta. The inclusion criteria were (1) patients undergoing hemodialysis 2-3 times a week, (2) adult patients aged $>18$ years with chronic medical diagnosis of chronic kidney disease, (3) patients had hemoglobin $(\mathrm{Hb})<10 \mathrm{~g} / \mathrm{dL},(4)$ patients had hemodialysis for more than 6 months, (5) patients can read and write, are able to communicate both verbal and non-verbal. Patients who had musculoskeletal disorders that affected their mobilization activities, patients with heart disorders, uncooperative patients, withdrawed patients at their own request and unwilling to respond were exclude.

Sampling technique in this activity was nonprobability sampling with consecutive sampling technique. Techniques to determine respondents to be included in the intervention group or control group using systematic random sampling that is by setting the odd number sequence as the intervention group and even serial number as the control group.

The implementation was done in Fatmawati Jakarta Hospital for one month starting from 13th March to 13th April 2017. The implementation was started by conducting pre-test related to patient's fatigue scale using Piper Fatique Scale measurement in both groups, 6 minutes intervention twice a week for two weeks (total of four interventions), examined the fatigue scale after 2 weeks in both groups (post-test), and complete the research reports. 


\section{Result}

Based on the research we found the results.

\section{Demographic characteristics}

Table 1. Distribution of Respondent Frequency Based on Characteristics of Age, Sex,

Old Hemodialysis and Hemoglobin Level at Fatmawati Hospital $(\mathrm{n} 1=\mathrm{n} 2=8)$

\begin{tabular}{|c|c|c|c|}
\hline No & Characteristics & $\begin{array}{c}\text { Frequency } \\
\text { Intervention Group (\%) }\end{array}$ & Frequency Control Group (\%) \\
\hline \multirow[t]{4}{*}{1.} & Age & & \\
\hline & $18-40$ years & $3(37,5)$ & $2(25,0)$ \\
\hline & $41-60$ years & $4(50,0)$ & $5(62,5)$ \\
\hline & $>61$ years & $1(12,5)$ & $1(12,5)$ \\
\hline \multirow[t]{3}{*}{2.} & Gender & & \\
\hline & Male & $6(75,0)$ & $5(62,5)$ \\
\hline & Female & $2(25,0)$ & $3(37,5)$ \\
\hline \multirow[t]{5}{*}{3.} & Hemodialysis Term & & \\
\hline & $6-12$ months & & \\
\hline & $13-24$ months & $1(12,5)$ & $1(12,5)$ \\
\hline & $>24$ months & $2(25,0)$ & $3(37,5)$ \\
\hline & & $5(62,5)$ & $4(50,0)$ \\
\hline \multirow[t]{4}{*}{4.} & Hemoglobin & & \\
\hline & Content & & \\
\hline & $<7 \mathrm{~g} / \mathrm{dL}$ & $3(37,5)$ & $2(25,0)$ \\
\hline & 7-10 g/dL & $5(62,5)$ & $6(75,0)$ \\
\hline
\end{tabular}

The analysis of age characteristic data in the intervention group showed the proportion of age 41-60 years with the highest number of $50 \%$. In the control group the proportion of age 41-60 years with the highest number of $62.5 \%$.

The analysis of sex characteristics data in the intervention group showed the proportion of male sex with the highest number was $75 \%$. In the control group the proportion of male sex with the highest number of $62.5 \%$.

The result of analysis of the long characteristic data undergoing hemodialysis in the intervention group showed that the proportion of $>24$ months with the highest number was $62,5 \%$. In the control group proportion $>24$ months with the highest number of $50 \%$.

Result of data analysis characterized hemoglobin level in the intervention group showed the proportion of $\mathrm{Hb} 7-10 \mathrm{~g} / \mathrm{dL}$ with the highest amount of $62,5 \%$. In the control group showed that the proportion of $\mathrm{Hb} 7-10 \mathrm{~g} / \mathrm{dL}$ with the highest amount of $75 \%$. 


\section{The Six-Minute Walking Exercise Intervention to Fatigue Rate}
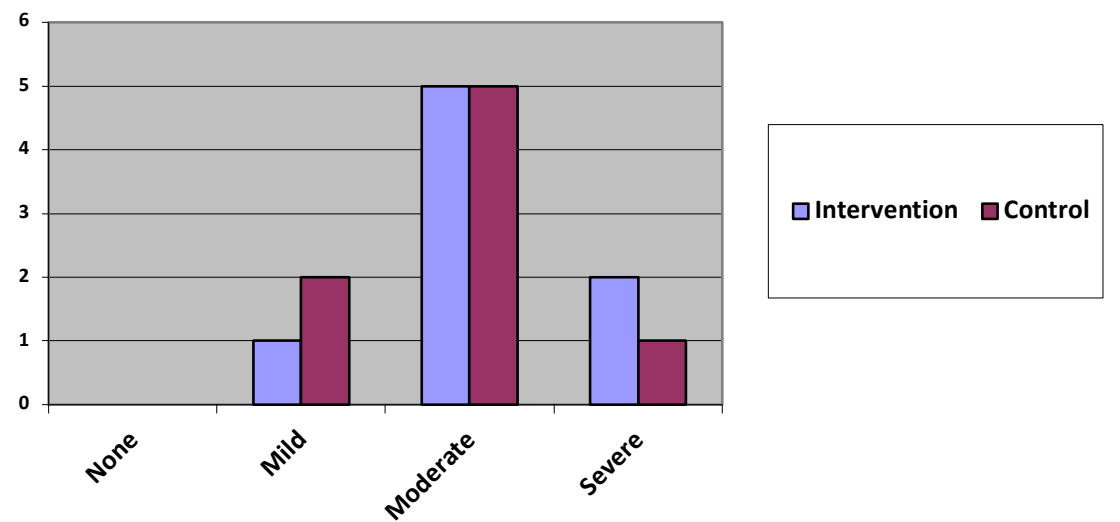

Figure 1.Distribution of Respondents by Fatigue Before the Six-Minute Walking (Pretest) Intervention at Fatmawati Hospital $(\mathrm{n} 1=\mathrm{n} 2=8)$

Figure 1 depicts the initial fatique levels in patients before the walking intervention is assessed using the Piper Fatique Scale instrument. The graph shows of the 16 respondents, all experienced fatigue $(0 \%)$ in both groups, intervention and control. In the intervention group, 1 respondent (12.5\%) experienced mild fatigue, 5 respondents $(62.5 \%)$ moderate fatigue and 2 respondents (25\%) fatigue. In the control group 2 respondents (25\%) experienced mild fatigue, 5 respondents $(62.5 \%)$ moderate fatigue and 1 respondent (12.5\%) fatigue.
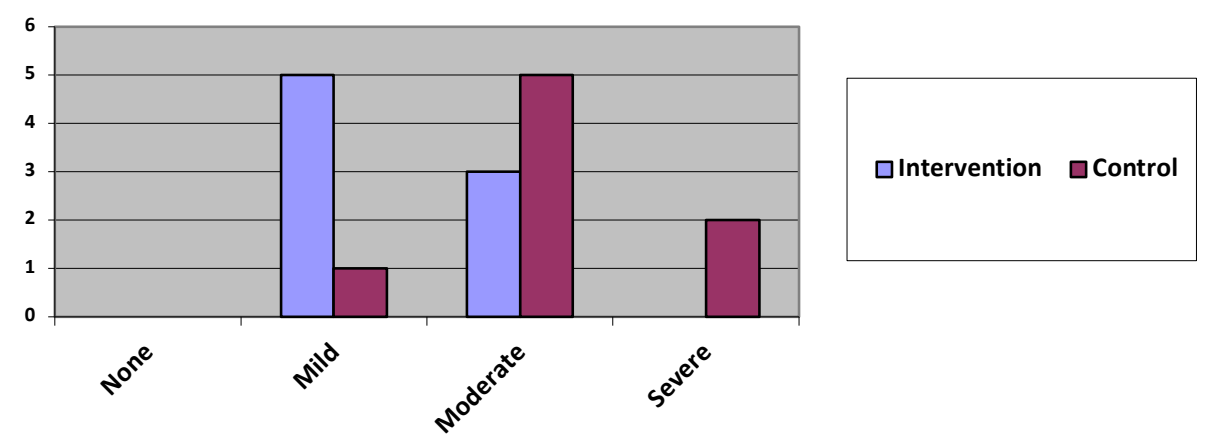

Figure 2. Distribution of Respondent Based on Fatique Level After the Six-Minute Walking (Posttest 4) Intervention at Fatmawati Hospital $(n 1=n 2=8)$

Figure 2 describes the patient's fatique level after six-minute walking nursing interventions were assessed using the Piper Fatique Scale instrument. The graph shows that of the 16 respondents, no one experience fatigue $(0 \%)$ in both groups, intervention and control. In the intervention group, there were 5 respondents (62.5\%) experiencing mild fatigue, 3 respondents $(37.5 \%)$ had moderate fatigue and no fatigue $(0 \%)$. In the control group, data obtained 1 respondent $(12.5 \%)$ experienced mild 
fatigue, 5 respondents $(62.5 \%)$ experienced moderate fatigue and 2 respondents $(25 \%)$ experienced severe fatigue.

Table 2. Distribution of Respondents Based on the Mean of Fatigue Levels after SixMinute Walking to 1 s.d to 4 in the Intervention Group at Fatmawati Hospital $(\mathrm{n} 1=\mathrm{n} 2=8)$

\begin{tabular}{lc}
\hline \multicolumn{1}{r}{ Fatique Level } & Characteristics \\
\hline Pre & $5,653 \pm 1,553$ \\
Post Intervention 1 & $5,324 \pm 1,512$ \\
Post Intervention 2 & $4,862 \pm 1,487$ \\
Post Intervention 3 & $2,713 \pm 1,389$ \\
Post Intervention 4 & $2,475 \pm 1,346$ \\
\hline
\end{tabular}

The result of fatigue level tassessment is a description of the subjective response of respondents. The average result of fatigue level in the intervention group in pre intervention shows the category of moderate fatigue level with a mean value of 5,653 . The decrease in mean values in the intervention group started at the first intervention of 5.324. In subsequent intervention, there is a decrease in mean and standard deviation so that after the fourth intervention the fatigue scale becomes a mild category of fatigue, with a mean value of 2.475 .

Table 3. Distribution of Respondents Based on the Mean of Fatigue Level between Intervention Groups Given Six-Minute Walking interval with Control Group at Fatmawati Hospital $(\mathrm{n} 1=\mathrm{n} 2=8)$

\begin{tabular}{lccc}
\hline \multirow{2}{*}{ Fatique Level } & \multicolumn{2}{c}{ Mean \pm SD } & \multirow{2}{*}{ Mean Difference } \\
\cline { 2 - 3 } & Pre & Post & \\
\hline Intervention Group & $5,653 \pm 1,553$ & $2,475 \pm 1,346$ & 3,178 \\
\hline Control Group & $5,327 \pm 1,511$ & $5,496 \pm 1,537$ & 0,169 \\
\hline
\end{tabular}

The results of the data analysis showed that in both intervention and control groups showed the mean of moderate fatigue level before the six-minute walking intervention with the value of 5.653 in the intervention group and 5.327 in the control group. The decrease in the mean and standard deviation in intervention group after giving six-minute walking intervention on mean final result with value equal to 2.475 with difference of mean difference between pre- and post- intervention equal to 3.178 and deviation standard value in intervention group before intervention from 1.553 to 1.346 .

This suggests that in the intervention group there was a decrease from the moderate fatigue category to the mild fatigue category. While in the control group, there was an increase of mean value from 5.327 to 5.496 with difference of 0.169 and 
for deviation standard there was an increase from 1.511 to 1.537 . This shows that in the control group there is no change of category, that is, the category of fatigue is good at pre and post six-minute walking intervention.

\section{Discussion}

Six-minute walking exercise is one of non-pharmacology intervention that can be applied in patients undergoing hemodialysis. This exercise overcomes the fatique in hemodialysis patients associated with psychological factors such as depression and anxiety that trigger is stress. Stress response enters the central nervous system, then the hypothalamus is released corticotrophin causing factor that stimulates the sympathetic nervous system to release norepinephrine which is a vasoconstrictor and result in smooth muscle contraction.

The six-minute walking exercises can decrease the fatique complaints by inhibiting the path above by activating the parasympathetic nervous system and manipulating the hypothalamus through concentration of the mind to reinforce the positive attitude so that the stress stimulus to the hypothalamus is reduced. In addition, six-minute walking exercises give the effect of muscle relaxation resulting in vasodilation of blood vessels that provide a calm and comfortable effect.

This exercise also lowers the fatique by lowering the tension physiologically, increasing muscle relaxation, decreasing anxiety so that vasodilation of blood vessels occurs. Systemic blood flow becomes smooth, the pulse becomes normal, the frequency of breathing becomes normal and reduces the evaporation process so that the patient becomes comfortable and the mind becomes quiet as a result of decreased activity of the reticular activating system (RAS) and increased brain stem activity, this can reduce the symptoms of fatique. ${ }^{6}$

The results showed an impact of six-minute walking exercises can decrease fatigue complaints (fatique) in hemodialysis patients. The decrease in fatigue resulted by six-minute walking exercises can increase blood flow in the muscle, increase the capillary amount, increase capillary surface area to increase urea and toxin transfer from tissue to vascular and then to dialyzer or hemodialysis machine. ${ }^{7}$

The results of another study showed that six-minute walking exercises can reduce fatigue complaints by increasing the stability of systolic and diastolic blood pressure. The cardiovascular system and the muscles used during the exercise were adapting. Adaptation results in the form of increased efficiency of cardiovascular system and active muscles. Adaptation will show many changes in the nerves, physical and biochemistry in the cardiovascular and muscle systems.

Walking exercises trigger stimulation of fibers in the skeletal muscles that includes sympathetic nervous responses. Sympathetic nerve responses include peripheral vascular vasoconstriction increasing cardiac muscle contractility, increasing heart rate and stabilizing blood pressure, thus improving cardiac output. Increased cardiac output is due to increased contractility of the heart muscle heart rate and blood flow along the working muscles. ${ }^{8}$

During exercise, muscle vessels dilate and blood flow increases, resulting in an increase in oxygen flow. At some levels, oxygen consumption is proportional to the energy released and all energy requirements are met through aerobic process. When muscles work has started very strongly, the aerobic resistance to energy savings can not keep up with the 
speed of its use. In such circumstances, phosphorylcreatin is still used for the re-synthesis of ATP (Adenosine triphosphate). Some ATP synthesis is met by using the energy released through the anaerobic decomposition of glucose into lactate. ${ }^{9}$

\section{Conclusion}

The results of six-minute walking exercises are proven to reduce the level of fatigue on patients undergoing hemodialysis. This is evidenced in the intervention group where there is a decrease in fatigue level from the category of moderate fatigue to mild fatigue. Thus, health service institutions are expected to implement and develop six-minute walking exercises as a part of their health education programs to reduce the level of fatigue of patients undergoing hemodialysis.

\section{Reference}

1. Smeltzer SC, Bare BG. Textbook of Medical Surgical Nursing. 11th ed. Philadelphia: Lipincott Williams \& Wilknis

2. International NANDA. Diagnosis Keperawatan: Definisi Dan Klarifikasi 2009 - 2011. Jakarta: EGC

3. O S, D., McCarthy G. An exploration of the relationship between Fatique and physical functioning in patinets with end stage renal disease receiving haemodialysis. J Clin Nurs. 16(11c):276 - 284.

4. McCann K, Boore J. Fatique in persons with renal failure who require manintenance haemodialysisi. J Adv Nurs. 32)5:1132-1142.

5. Jazi H, Mansooreh A. Effect of walking on pain from muscle cramps in haemodialysis patients. Int Sport J. 13:161-169.

6. Malagoni A. M. et al. Acute and long-term effects of an exercise program for dialysis patients prescribed in hospital and performed at home. J Nephrol. 21(6):871-878.

7. Maja B, Cuk I, Bojan L. Six Minute Walk Test in Renal Failure Patients: Representative Results. In: Performance Anaysis and Perceived Fatique Predictors: PLOS ONE. doi:10.1371/journal.pone.0150414.

8. Pollentier B, Irons S. Examination of The Six Minute Walk Test to Determine Functional Capacity in People with Chronic Kidney Disease. Kidney Inter. Suppl. 2:279-334. doi:10.1038/kisup.2012.40

9. A.C. G, Hall JE. Buku Ajar Fisiologi Kedokteran. Edisi. 9. 